\title{
Endovascular treatment of late coronary-subclavian steal syndrome
}

\author{
Gianluca Faggioli, MD, ${ }^{\mathrm{a}}$ Rodolfo Pini, MD, ${ }^{\mathrm{a}}$ Alberto Cremonesi, MD, ${ }^{\mathrm{b}}$ Chiara Grattoni, MD, \\ Matteo Longhi, MD, ${ }^{a}$ Raffaella Mauro, MD, ${ }^{a}$ Fausto Castriota, MD, ${ }^{b}$ and Andrea Stella, MD
}

\begin{abstract}
Objective: Coronary-subclavian steal syndrome (CSSS) is a rare cause of myocardial ischemia subsequent to stenosis or occlusion of the subclavian artery (SA) proximal to internal thoracic artery (ITA) coronary bypass. Only single cases have been reported in published studies to date. We report a significant series of patients with late CSSS treated through an endovascular approach.
\end{abstract}

\begin{abstract}
Methods: We reviewed a series of consecutive patients treated for CSSS. The clinical, anatomic, and technical characteristics of the procedures were considered. Follow-up was performed through clinical and laboratory (electrocardiography, echocardiography, duplex ultrasonography) evaluations.

Results: From January 2005 to March 2013, 10 patients with CSSS were treated; 7 had stable and 3 unstable angina. Of the 10 patients, 8 had left SA stenosis (6 ostial to the origin and 2 in the middle segment), 1 had proximal occlusion of the left SA, and 1 had stenosis in the innominate artery (proximally to a right internal thoracic artery). Arterial access was at the brachial artery through surgical exposure $(n=6)$, or radial artery percutaneously $(n=3)$. In 1 case of proximal occlusion of the left SA, simultaneous femoral and percutaneous radial access was necessary. Predilatation of the stenotic lesion was performed in 6. Balloon expandable stents were used in 7 patients with proximal ostial stenosis or occlusion and self-expandable stents in 2 with nonostial lesions. In 1 other patient with proximal heavy calcified stenosis, cutting-balloon predilatation was performed, resulting in dissection of the SA and occlusion of the ITA graft; blood flow was restored in the left upper arm and myocardium by adjunctive dilatation of the SA and endovascular coronary revascularization. No patients developed angina during the follow-up period ( $15 \pm 7$ months).
\end{abstract}

Conclusions: A tailored endovascular approach can be used to treat CSSS. However, the occurrence of potentially lethal complications is possible and needs prompt correction. (J Thorac Cardiovasc Surg 2014;148:2112-6)

Coronary-subclavian steal syndrome (CSSS) is a rare cause of myocardial ischemia subsequent to occlusive disease of the subclavian artery (SA) proximally to an internal thoracic artery (ITA) coronary bypass. ${ }^{1}$ If SA occlusive disease, possibly leading to CSSS, is detected before the coronary bypass procedure, the surgical approach to myocardial revascularization can be modified or the stenosis corrected beforehand or simultaneously. ${ }^{2-4}$ However, postoperative CSSS will develop in about $1 \%$ to $4 \%$ of patients who have undergone coronary artery bypass grafting (CABG) with an ITA graft, ${ }^{2,5}$ with its late incidence undefined in published studies. The onset of CSSS some years after coronary revascularization has been sporadically reported through single case reports. ${ }^{6-11}$ Treatment of CSSS aims to correct the SA stenosis, which

From the Department of Vascular Surgery, ${ }^{\text {a }}$ University of Bologna, Policlinico S. Orsola-Malpighi, Bologna, Italy; and Interventional Cardiovascular Unit, ${ }^{b}$ GVM Care and Research, Maria Cecilia Hospital, Cotignola, Ravenna, Italy.

Disclosures: Authors have nothing to disclose with regard to commercial support.

Received for publication March 4, 2014; revisions received April 11, 2014; accepted for publication May 16, 2014; available ahead of print June 18, 2014.

Address for reprints: Rodolfo Pini, MD, Department of Vascular Surgery, University of Bologna, Policlinico S. Orsola-Malphighi, via Massarenti 9, Bologna 40138, Italy (E-mail: rudypini@gmail.com).

$0022-5223 / \$ 36.00$

Copyright $($ C 2014 by The American Association for Thoracic Surgery

http://dx.doi.org/10.1016/j.jtcvs.2014.05.036 is usually severe and associated with heavy calcifications. The technique of choice is yet to be defined; however, an endovascular approach could be advantageous in terms of invasiveness. Surgical extra-anatomic bypass (subclavian-subclavian or carotid-subclavian) can be a valuable alternative. ${ }^{12,13}$

The aim of the present study was to review our experience in the treatment of late CSSS.

\section{METHODS}

\section{Study Design and CSSS Diagnosis}

We retrospectively reviewed, from January 2005 to July 2013, the experience of a vascular surgery center (S. Orsola-Malpighi, Bologna, Italy) and cardiologic interventional center (Maria Cecilia Hospital, Cotignola, Ravenna, Italy) in the treatment of late CSSS. All patients gave their informed consent for the procedure. The study was performed according to the rules of the ethical review boards of our institutions.

All patients who had undergone CABG with an ITA graft and had clinical symptoms of angina were evaluated by coronarography and supra-aortic vessel angiography. Computed tomography angiography was also performed in some instances. Hemodynamic stenosis proximal to the ITA was defined as a trans-stenosis pressure reduction of $30 \mathrm{~mm} \mathrm{Hg} .^{3,12,13}$ The indication to treat the stenosis was the simultaneous presence of symptoms of myocardial ischemia and SA hemodynamic stenosis proximal to the ITA. ${ }^{12}$

\section{Patients}

The patients' clinical characteristics, interval from CABG to the new angina symptoms, type of myocardial symptoms, technical aspects of the 

Abbreviations and Acronyms
$\mathrm{CABG}=$ coronary artery bypass grafting
CSSS = coronary-subclavian steal syndrome
ITA $=$ internal thoracic artery
SA $=$ subclavian artery

revascularization procedure, and perioperative (30-day) outcomes were entered into a dedicated database. The stenosis was categorized as ostial (in the SA or innominate artery) or located in the middle segment of the vessel proximal to the ITA.

The clinical characteristics included the following: age, gender, hypertension (presence of systolic blood pressure $>140$ and/or diastolic blood pressure $>90 \mathrm{~mm} \mathrm{Hg}$ or specific therapy), dyslipidemia (total cholesterol $>200 \mathrm{mg} / \mathrm{dL}$ or low-density lipoprotein $>120 \mathrm{mg} / \mathrm{dL}$ or specific therapy), diabetes mellitus (prediagnosed in therapy with oral hypoglycemic drugs or with insulin), current smoking, chronic obstructive pulmonary disease (defined as chronic bronchitis or emphysema), and chronic renal failure (glomerular filtration rate $<60 \mathrm{~mL} / \mathrm{min}$ ). Ongoing therapy with antiplatelet drugs (single or double), $\beta$-blockers, anticoagulant agents, and statins was also considered.

The clinical and electrocardiographic findings of new myocardial ischemic symptoms and recurrence of proximal SA disease by duplex ultrasound examination were considered in the follow-up period.

\section{Revascularization Procedures}

All procedures were performed with the patients under local anesthesia and systemic heparinization. The access site varied according to the location and type of occlusive disease (stenosis vs occlusion). In the vascular surgery department, surgical exposure of the brachial artery was the approach of choice. In the interventional coronary unit, percutaneous access was the approach of choice. Predilatation was performed when necessary to cross the stenosis with the stent. Balloon expandable stents were used for the ostial lesions and self-expandable for lesions located in the middle segment of the vessels.

The perioperative results included "technical success," defined as treatment resulting in $<30 \%$ residual stenosis, myocardial or neurologic ischemic events, and access complications (hematoma with the necessity for blood transfusion or surgical revision).

\section{RESULTS}

\section{General Characteristics}

From January 2005 to March 2013, 10 patients with CSSS were treated: 6 in the vascular surgery department and 4 in the cardiologic interventional unit. The mean interval from CABG to the development of angina symptoms was $9.0 \pm$ 8.4 years. The patients' clinical characteristics are reported in Table 1. Because all patients were referred from other institutions, we could not assess the incidence of CSSS. Of the 10 patients, 7 had an ostial SA lesion (4 stenosis and 3 occlusion), 2 had stenosis of the middle segment of the left SA, and 1 had stenosis in the middle segment of the innominate artery proximally to a right ITA CABG (Table 2). All patients with unstable angina had total ostial SA occlusion and vice versa.

\section{Vascular Access}

Surgical left brachial artery exposure. In all 6 patients treated in the vascular surgery department, surgical
TABLE 1. Patient characteristics

\begin{tabular}{lc}
\hline \multicolumn{1}{c}{ Variable } & $\mathbf{n}(\%)$ \\
\hline Clinical characteristics & \\
Male gender & $7(70)$ \\
Age (y) & $73.3 \pm 7.6$ \\
Hypertension & $10(100)$ \\
Dyslipidemia & $10(100)$ \\
COPD & $3(30)$ \\
CRF & $3(30)$ \\
Diabetes & $6(60)$ \\
Smoke & $3(30)$ \\
Statins & $8(80)$ \\
Double antiplatelet agents & $10(100)$ \\
$\beta$-blockers & $8(80)$ \\
Interval from CABG (y) & $9 \pm 8.4$ \\
Type of symptoms & \\
Stable angina & $7(70)$ \\
Unstable angina & $3(30)$ \\
\hline
\end{tabular}

Data presented as $\mathrm{n}(\%)$ or mean \pm standard deviation. COPD, Chronic obstructive pulmonary disease; $C R F$, chronic renal failure; $C A B G$, coronary artery bypass grafting.

exposure of the left brachial artery was performed. This approach allows one to cross the lesion with the angiographic catheter in the presence of stenosis and to have sufficient "pushability" through a 6F, 10-cm, introducer (Cordis, Waterloo, Belgium) in the presence of occlusion to cross it with the guidewire (Terumo, Tokyo, Japan).

Percutaneous radial access. Percutaneous radial access was used in 3 patients treated in the cardiologic interventional unit through a $6 \mathrm{~F}$ sheath (Cordis, Waterloo, Belgium), 2 with stenosis of the middle segment of the SA and 1 of the innominate artery.

Simultaneous percutaneous femoral and radial access. In the case of ostial occlusion of the left SA, simultaneous right femoral (7F sheath Cordis, Waterloo, Belgium) and left radial (6F sheath, Cordis) percutaneous access was performed.

\section{Stenosis Crossing, Predilatation, and Stenting}

The stenoses were crossed by a 0.035 -in. floppy wire (Terumo, Tokyo, Japan) in 4 patients; in 3 patients with

TABLE 2. Anatomic features

\begin{tabular}{ll}
\hline \multicolumn{1}{c}{ Variable } & $\mathbf{n}(\%)$ \\
\hline Lesion type & $7(70)$ \\
Stenosis & $4(57)$ \\
$\quad$ Subocclusive & $3(43)$ \\
$\quad$ Nonsubocclusive & $3(30)$ \\
Obstruction & \\
Stenosis location & $4(57)$ \\
Ostium of left SA & $2(29)$ \\
Middle segment of SA & $1(14)$ \\
Innominate artery & \\
Obstruction location & $3(100)$ \\
Ostium of left SA &
\end{tabular}




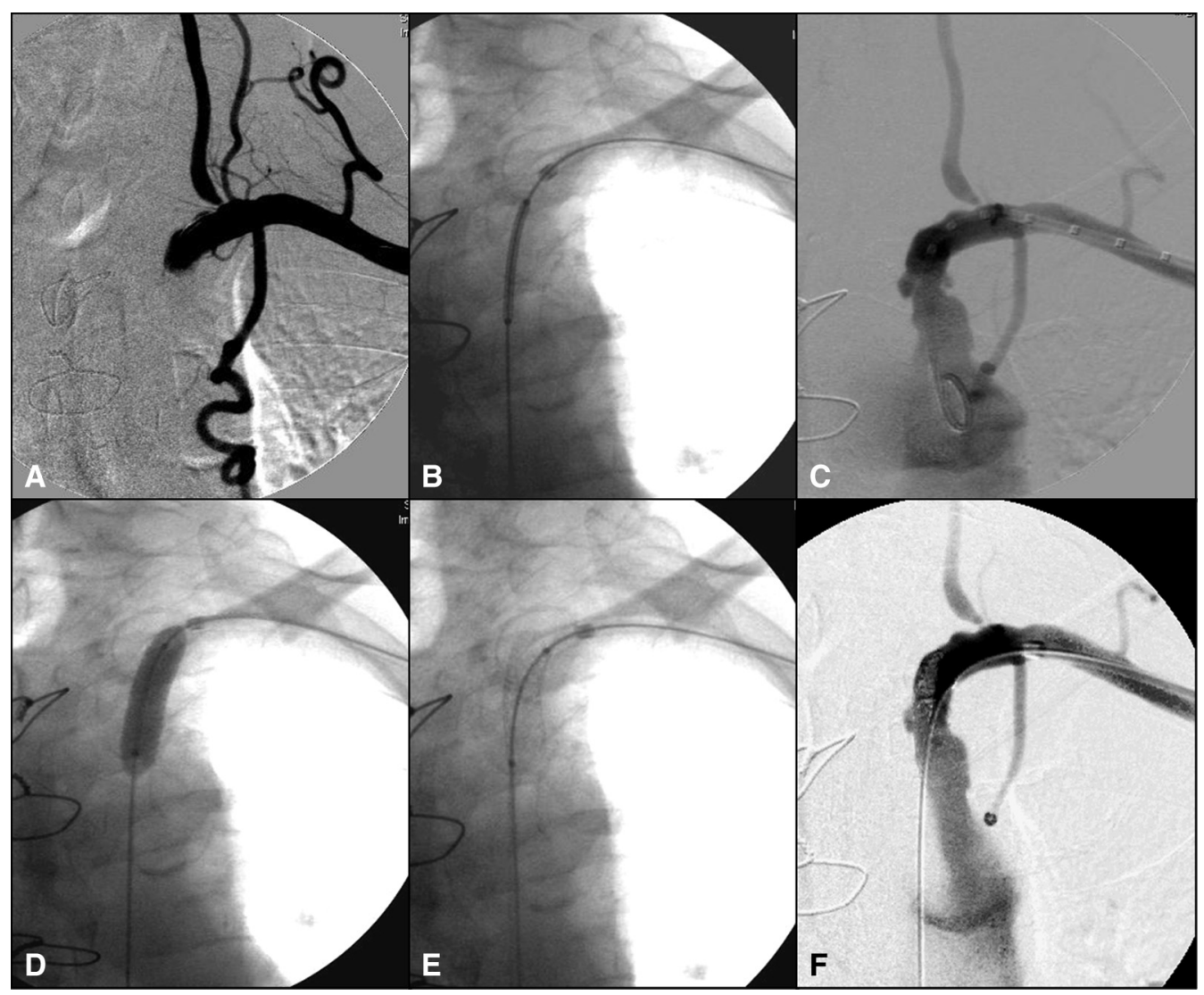

FIGURE 1. Focal occlusion of middle segment of subclavian artery. A, Preoperative angiographic view; B, view of predilatation; C-E, stent deployment; and $\mathrm{F}$, final angiographic view.

subocclusive stenosis and 3 with occlusion, a stiff 0.018-in. (V18, Boston Scientific, Natick, Mass) wire was used (Figure 1). Predilatation was performed in 6 cases with a balloon catheter, ranging from $2.5 \times 4.0 \mathrm{~mm}$ to $5.0 \times 4.0$ $\mathrm{mm}$. In 1 patient with a heavily calcified ostial SA stenosis, dilatation with cutting balloon $(4.0 \times 15 \mathrm{~mm}$, Flextome Cutting Balloon, Natick, Mass) was performed and was complicated by SA spiral dissection and ITA occlusion (Figure 2). The SA dissection was resolved with prolonged ballooning of the SA, allowing reperfusion of the upper left arm. Also, an endovascular coronary revascularization was performed to maximize the myocardial perfusion.

The stent type was chosen according to the type of stenosis. A balloon expandable stent was placed in the 3 ostial stenoses and in the 3 ostial occlusions (Table 3) to achieve accurate dilatation and avoid protrusion of the stent in the aortic arch. A self-expandable stent was placed in the 2 stenosis of the middle segment of the SA and in the stenosis of the innominate artery.

\section{Outcome and Follow-up}

Technical success was achieved in 9 patients. The 1 patient with dissection of the SA did not have any myocardial complications; however, the ITA graft was lost, and the case should be considered a technical failure. No myocardial or neurologic complications developed. The patient with SA dissection and ITA occlusion had no myocardial sequelae. One patient with surgical left brachial artery access developed a hematoma that required surgical revision on postoperative day 1. At a mean follow-up of $14.4 \pm 8.7$ months, all patients were asymptomatic for new angina symptoms, with no signs of restenosis at duplex ultrasonography, either direct (in the cases of stenting of the middle SA) or indirect (through measurement of the flow distal to an ostial lesion).

\section{DISCUSSION}

The number of patients with late CSSS reported in the present study is the most significant published to date. However, the frequency of CSSS could not be assessed, because all the patients were referred from other hospitals. Thus, we have combined the patients treated in 2 units (vascular surgery unit and interventional cardiologic unit), describing 2 different approaches to the same problem. In patients treated by the vascular surgeons, a minimal brachial artery surgical exposure was chosen for access to have greater "pushability" of the wire for crossing the 


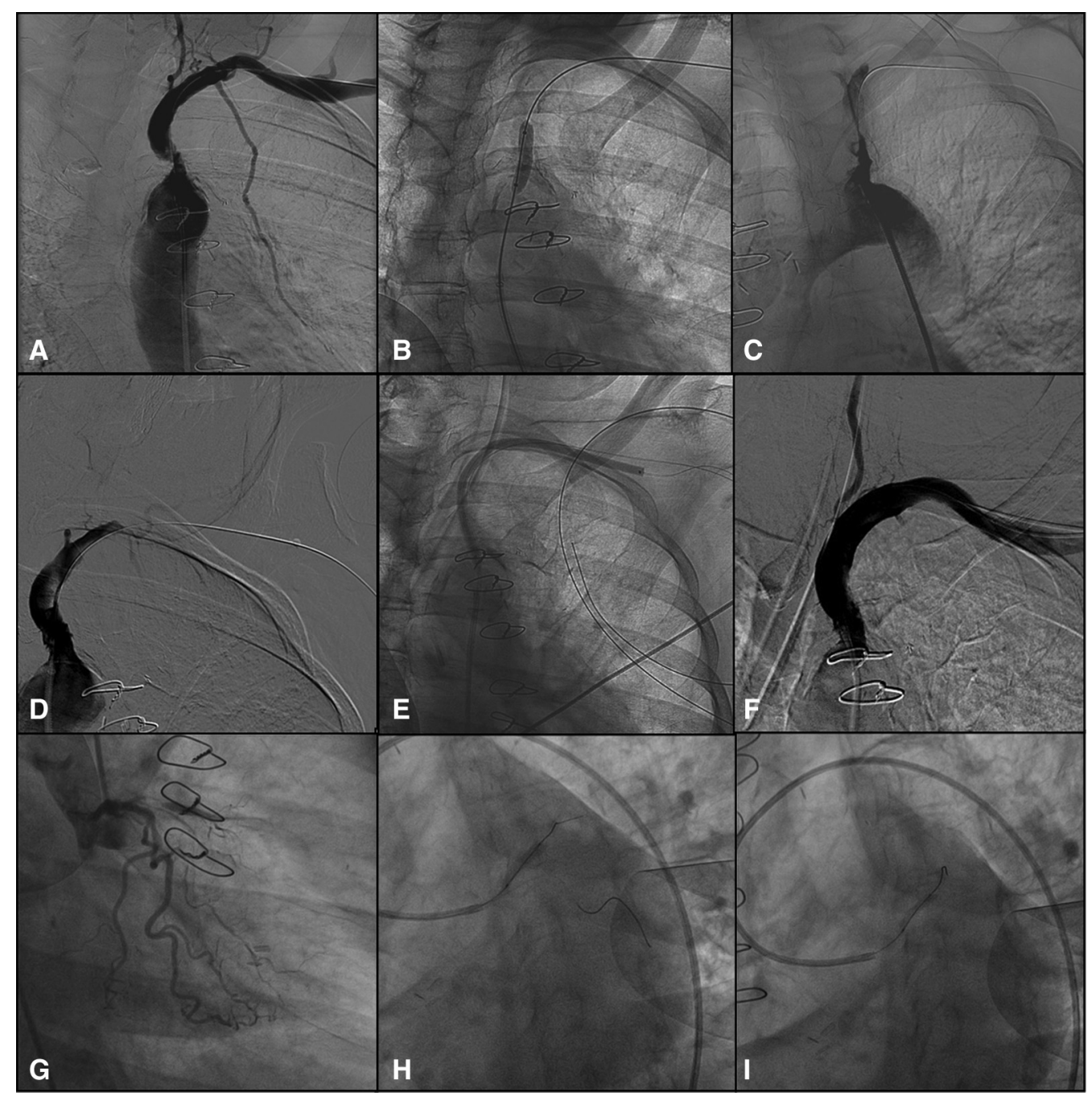

FIGURE 2. Treated subclavian stenosis complicated by arterial dissection. A, Preoperative angiographic view; B, predilatation with cutting balloon; $\mathrm{C}$ and D, subclavian artery dissection; E and F, subclavian artery dilatation, resolution of the dissection, and occlusion of the internal thoracic artery; and G-I, coronary revascularization.

lesion. Surgical exposure of the vessel is easily performed by experienced vascular surgeons, with minimal potential for complications. The radial approach could be advantageous for skilled cardiologists, but when inadequate, the percutaneous upper arm approach might be troublesome in some patients.

Balloon expandable stents were used in the presence of an ostial lesion; in those cases, high deployment precision is necessary to avoid inadvertent protrusion of the stent in the aortic arch or distal slipping. However, a selfexpandable stent was chosen for long stenoses of the medial vessels segments, given the minor radial force necessary and the low risk of misplacement. Balloon-expandable stents were generally chosen 1 or $2 \mathrm{~mm}$ smaller than the native arterial diameter, in consideration of the severe stenosis present and the stent overdilatation caused by the balloon. In contrast, self-expandable stents were 1 to 2 mm larger than the artery diameter because of their greater conformability.

Despite the overall satisfactory results obtained in the present series, a major complication occurred when the predilatation with a cutting balloon led to a dissection of the SA and occlusion of the ITA. Although the complication was successfully resolved with prolonged SA ballooning and new coronary endovascular revascularization, with no myocardial or neurological sequelae, it is a potentially lethal event. Thus, its occurrence must be considered when submitting patients with CSSS to endovascular treatment. The cutting ballooning should therefore be used cautiously in SAs with heavy calcified stenoses.

Only case reports have been published about late CSSS and its treatment. Most have referred to endovascular approaches, with good results in the short to medium term. In their report, Song and colleagues ${ }^{14}$ compared 
TABLE 3. Material characteristics

\begin{tabular}{|c|c|c|}
\hline Characteristic & Lesion anatomy & n $(\%)$ \\
\hline \multicolumn{3}{|l|}{ Stent type } \\
\hline \multirow[t]{3}{*}{ Self expandable } & & $3(30)$ \\
\hline & Stenosis of innominate artery & 1 \\
\hline & Stenosis of middle segment of SA & 2 \\
\hline \multirow[t]{3}{*}{ Balloon expandable } & & $6(60)$ \\
\hline & Subocclusive ostial stenosis & 3 \\
\hline & Ostial obstruction & 3 \\
\hline Sheath type & & 10 \\
\hline \multicolumn{3}{|l|}{$6 \mathrm{~F}$} \\
\hline \multirow[t]{3}{*}{ Percutaneous radial access } & & $3(30)$ \\
\hline & Stenosis of innominate artery & 1 \\
\hline & Stenosis of middle segment of SA & 2 \\
\hline \multirow{2}{*}{ Percutaneous femoral access } & & $1(10)$ \\
\hline & Ostial occlusion & 1 \\
\hline \multirow[t]{3}{*}{ Surgical brachial access } & & $6(60)$ \\
\hline & Ostial stenosis of SA & 4 \\
\hline & Ostial occlusion of SA & 2 \\
\hline \multicolumn{3}{|l|}{$7 \mathrm{~F}$} \\
\hline \multirow[t]{2}{*}{ Percutaneous femoral access } & & $1(10)$ \\
\hline & Ostial occlusion & 1 \\
\hline \multicolumn{3}{|l|}{ Wire type } \\
\hline \multirow[t]{2}{*}{ Floppy, 0.035 in. } & & $4(40)$ \\
\hline & Nonsubocclusive stenosis & 4 \\
\hline \multirow[t]{3}{*}{ Stiff, $0.018 \mathrm{in.}$} & & $6(60)$ \\
\hline & Subocclusive stenosis & 3 \\
\hline & Ostial obstruction & 3 \\
\hline \multirow[t]{3}{*}{ Predilatation } & & $6(60)$ \\
\hline & Subocclusive stenosis & $5 *$ \\
\hline & Ostial obstruction & $1 \dagger$ \\
\hline
\end{tabular}

SA, Subclavian artery. *Balloon diameter, $2.5 \times 40$ to $5.0 \times 40 \mathrm{~mm}$. †Cutting balloon, $4.0 \times 15 \mathrm{~mm}$.

endovascular and surgical revascularization for subclavian steal syndrome in patients without ITA CABG. Both endovascular stenting $(\mathrm{n}=148)$ and extrathoracic surgical bypass $(\mathrm{n}=104)$ were shown to be safe and effective in the short to medium term. However, surgical bypass has been more durable, with a 5-year primary patency for endovascular stenting versus extrathoracic surgical bypass of $67 \%$ versus $95 \%$ and a cumulative secondary 10 -year patency of $64 \%$ versus $94 \%$, respectively.

The surgical approach can be an alternative to endovascular treatment; however, no studies have compared these 2 techniques for CSSS. Surgical subclavian revascularization can be performed using several methods, such as subclavian to subclavian bypass ${ }^{6}$ or carotid to subclavian bypass. When stenosis or occlusion of the left SA has been identified before CABG, the surgical approach of the latter can be modified with the performance of extrathoracic bypass grafts or anastomosis of the saphenous vein graft directly to the aorta, using the ITA as a bypass graft from the aorta to the subclavian vein. ${ }^{15}$

\section{CONCLUSIONS}

CSSS is a severe pathologic entity associated with extensive atherosclerotic involvement. ${ }^{9}$ To restore direct flow in the SA is the key point to preserving the coronary perfusion provided by the ITA, and the endovascular approach is effective, durable, and generally safe. However, the risk of severe complications should not be underestimated, particularly in the case of calcific lesions.

\section{References}

1. Gloger V. Coronary-subclavian steal syndrome, a complication following surgical revascularization of myocardium. Vnitr Lek. 2012;58:871-4.

2. Wisneski AD, Beyer AT, Shunk KA. An unusual case of bilateral subclavian-carotid artery graft occlusion with coronary steal syndrome managed in the cath lab. J Invasive Cardiol. 2013;25:E14-6.

3. Bindea D, Todoran A, Mihai T, Ober C, Iancu A. Coronary-subclavian steal syndrome treated with carotid to subclavian artery by-pass. Chirurgia (Bucur). 2013;108:264-7.

4. Dieter RS. Approach to subclavian artery stenosis prior to or after coronary bypass with the LIMA conduit: reply. J Endovasc Ther. 2012;19:461-3.

5. Mangialavori G, Ballo P, Michelagnoli S, Ercolini L, Barbanti E, Passuello F, et al. Subclavian steal syndrome presenting as recurrent pulmonary oedema associated with acute left ventricular diastolic dysfunction. Cardiovasc Revasc Med. 2013;14:45-8.

6. Pedro PG, Carôla B, Conduto R, Barão I, Ferreira RC, Magalhães MP. A case of coronary-vertebral subclavian steal syndrome. Rev Port Cardiol. 2013;32:443-5.

7. Schatzl S, Karnik R, Gattermeier M. Coronary subclavian steal syndrome: an extracoronary cause of acute coronary syndrome. Wien Klin Wochenschr. 2013;125:437-8.

8. Tariq S, Tuladhar S, Wingfield E, Poblete H. Coronary subclavian steal syndrome unamenable to angioplasty successfully managed with subclavian-subclavian bypass. Case Rep Vasc Med. 2012;2012:784231.

9. Mandak J, Lojik M, Tuna M, Chek JL. Coronary subclavian steal syndrome causing acute myocardial infarction in a patient undergoing coronary-artery bypass grafting. Case Rep Med. 2012;2012:798356.

10. Tan CS, Fintelmann F, Joe J, Ganguli S, Wu S. Coronary-subclavian steal syndrome in a hemodialysis patient: a case report and review of literature. Semin Dial. 2013;26:E42-6.

11. Tanaka A, Sakakibara M, Okada K, Jinno Y, Ishii H, Murohara T. Coronary subclavian steal from a left internal thoracic artery coronary bypass graft due to ipsilateral subclavian artery stenosis and an arteriovenous graft in a hemodialysis patient with left vertebral artery occlusion. Intern Med. 2013;52:1195-8.

12. John A, Hofmann S, Ostowar A, Ferdosi A, Warnecke H. Reversal of flow in the mammary artery to treat subclavian steal syndrome in conjunction with coronary bypass surgery. Ann Thorac Surg. 2011;91:283-5.

13. Karakulak UN, Kose IC, Evranos B, Okutucu S, Hazirolan T, Aytemir K, et al. Multimodality imaging of coronary-subclavian-vertebral steal syndrome. Circulation. 2012; 125:e241-3.

14. Song L, Zhang J, Li J, Gu Y, Yu H, Chen B, et al. Endovascular stenting vs. extrathoracic surgical bypass for symptomatic subclavian steal syndrome. J Endovasc Ther. 2012;19:44-51.

15. Özkan B, Açar G, Çakalagaoglu KC, Koksal C, Esen AM. A new form of coronary subclavian steal syndrome: a saphenous vein graft-coronary-subclavian unidirectional steal syndrome. Heart Surg Forum. 2012;15:210-1. 